\title{
Millimeter-Wave Textile Antenna for On-Body RF Energy Harvesting in Future 5G Networks
}

\author{
Mahmoud Wagih, Student Member, IEEE, Alex S. Weddell, Member, IEEE, Steve Beeby Senior Member, IEEE
}

School of Electronics and Computer Science, University of Southampton

\{mahm1g15, asw, spb\}@ecs.soton.ac.uk

\begin{abstract}
Millimeter-Wave (mmWave) bands will be a key part of future 5G networks, with the 26 and $28 \mathrm{GHz}$ bands being introduced first. The wide bandwidth aims to solve traffic-related issues. The projected high base-station density, highly directive transmitters, and the wide bandwidth make it a very promising RF energy harvesting (RFEH) source. Broadband antennas are necessary to harvest power efficiently from the full spectrum. This work presents the first antenna on textile for wearable ambient RFEH in the $26 \mathrm{GHz}$ and $28 \mathrm{GHz}$ bands. The antenna has an impedance bandwidth from 20 to $30 \mathrm{GHz}$, and exhibits a peak on-body gain of $7 \mathrm{~dB}$ with an omnidirectional radiation pattern for capturing ambient RF energy. The radiation efficiency on- and off-body was observed to be at least $40 \%$ and $60 \%$ respectively, between 24 and $30 \mathrm{GHz}$. A two-line microstrip dielectric characterization of the textile substrate in the mmWave band has been performed. The antenna has been fabricated on a $310 \mu \mathrm{m}$ woven polyester substrate using etched ultra-thin Polyimide copper laminates with a minimum feature size of $150 \mu \mathrm{m}$. A high robustness against human proximity has been demonstrated with a stable bandwidth and improved gain.
\end{abstract}

Index Terms-millimeter-Wave, RF Energy Harvesting, 5G, Antenna, Electronic Textiles, Internet of Things (IoT)

\section{INTRODUCTION}

The millimeter-wave (mmWave) band represents a major part of future $5 \mathrm{G}$ networks, where the broad spectrum enables mitigation of traffic related limitations in conventional cellular networks. Key features of future mmWave networks are the wide-spectrum, and the higher density of small base-stations [1]. However, at mmWave frequencies, waves are absorbed by air gases and water droplets, and their ability to penetrate obstacles is reduced [2]. Highly directional phased antenna arrays and beam-forming represent methods of overcoming the significantly higher propagation losses in the proposed bands.

Radio frequency energy harvesting (RFEH) is an increasingly popular method of powering low-power devices; potentially enabling battery-less operation of sensing and computing nodes at the network edge. The large transmitter antenna arrays, and the more-dense base-stations make future mmWave networks an attractive RFEH source due to the improved energy coverage [1]. Furthermore, It has been reported that $\mathrm{RFEH}$ in the mmWave bands is more efficient than existing sub-6 GHz cellular networks [1]. Additionally, RFEH,

This work was supported by the European Commission through the project EnABLES: European Infrastructure Powering the Internet of Things, funded under H2020-EU.1.4.1.2 and in part by the Engineering and Physical Sciences Research Council through the project Novel Manufacturing Methods for Functional Electronic Textiles under Grant EPSRC EP/M015149/1.
Wireless Power Transfer (WPT) and Simultaneous Wireless Information and Power Transfer (SWIPT) are expected to coexist in future $5 \mathrm{G}$ networks in the mmWave bands; due to the significantly broader spectrum [3].

Rectifying antennas (rectennas) are the key component in an RFEH or WPT system. Planar patch rectenna arrays [4], [5], and a substrate-integrated waveguide (SIW) rectenna [6] have previously been presented for the $24 \mathrm{GHz}$ Industrial Scientific Medical (ISM) band, with a $0.6 \mathrm{~V}$ DC output from $10 \mathrm{~mW}$ of incident mmWave power [4].

Electronic textiles have been previously characterised and developed for mmWave applications [7], [8]. A $60 \mathrm{GHz}$ antenna array, of $40 \%$ radiation efficiency has been previously fabricated using laser ablated copper foil, on a poly-cotton substrate for body centric communication in the $60 \mathrm{GHz}$ license-exempt band [8]. mmWave SIWs have been presented using woven rigid threads [9].

In this work, a textile-based antenna fabricated using etched Polyimide copper laminates, optimized for operation on-body, is presented for energy harvesting in the $26 \mathrm{GHz}$ and $28 \mathrm{GHz}$ $5 \mathrm{G}$ licensed bands. This is the first ultra-wide band (UWB) textile antenna covering the full licensed $5 \mathrm{G}$ mmWave spectrum. A novel design approach has been utilized to increase the antenna's gain on-body. Higher gain [5], and radiation efficiency [8] has been achieved compared to previous flexible millimeter-wave antennas.

\section{Antenna DeSIGN AND FABRICATION}

The proposed antenna is based on the UWB antipodal Vivaldi antenna [10], with a circular curve to improve the bandwidth. The main aim of the design process has been to prioritize maximizing the radiation efficiency by minimizing the radiator size. Antennas of large radiating elements, such as patches, exhibit higher dielectric losses and consequently lower radiation efficiency. The antenna and dimensions are shown in Fig. 1.

Among the candidates for the antenna's fabrication methods are screen and inkjet-printing [11], woven or adhered conductive textiles and copper foils [8], [12], as well as etched copper laminates [13]. A high resolution process, producing smooth homogeneous conductors, is required to minimize manufacturing imperfections, as the feature size of mmWave antennas is commonly less than $1 \mathrm{~mm}$. Conductor printing has been excluded due to the high thickness of the required 


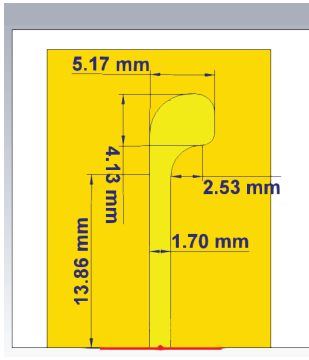

(a)

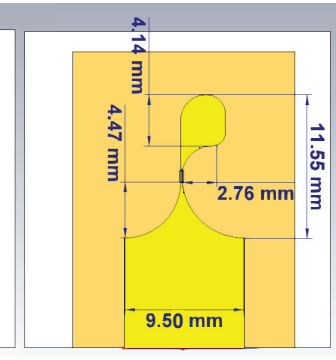

(b)

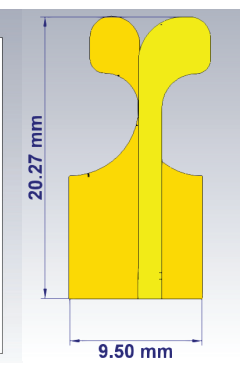

(c)
Fig. 1. Proposed antenna and dimensions. A: top layer, b: bottom layer, c: asymmetric antipodal alignment of both sides

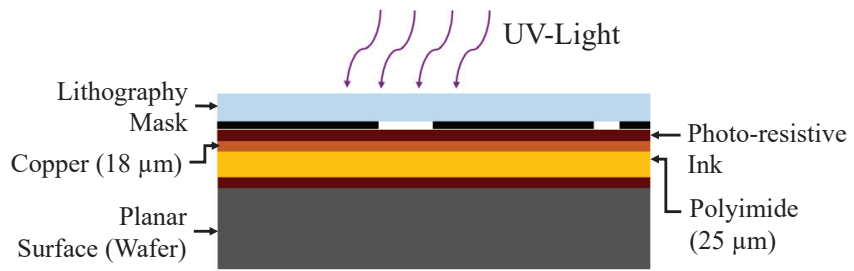

(a)

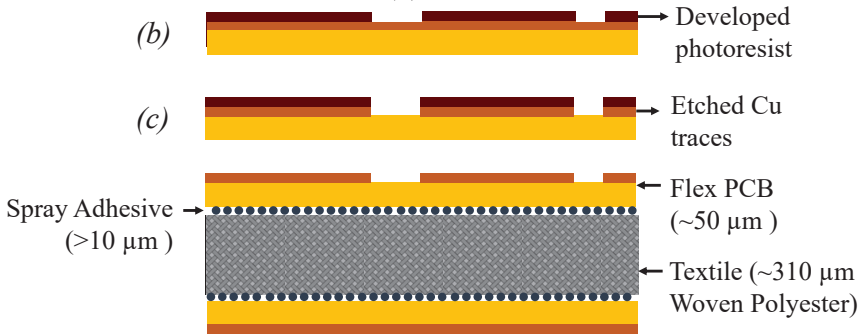

(d)

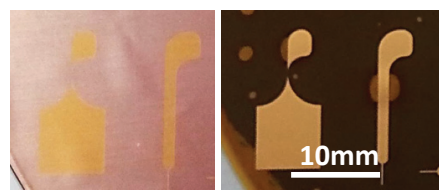

(e) (f)

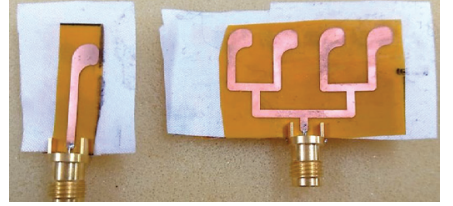

(g)

Fig. 2. Antenna fabrication process and photographs. (a): cross-section of structure during UV exposure, (b): cross-section before etching (c): crosssection after copper etching, (d): fully assembled structure on textile (e): photograph of $b$, (f): photograph of $\mathrm{c},(\mathrm{g})$ : photograph of $\mathrm{d}$ with a $1.85 \mathrm{~mm}$ test connector: single element (left) and $4 \times 1$ array (right).

interface layer [14], resulting in higher dielectric losses and reduced antenna efficiency.

Ultra-thin Polyimide copper laminates exhibit improved flexibility compared to copper foils, and lower thickness compared to printed interface layers; minimizing dielectric losses. The copper laminates are fabricated using a standard photolithography process, with the track's pattern plotted on

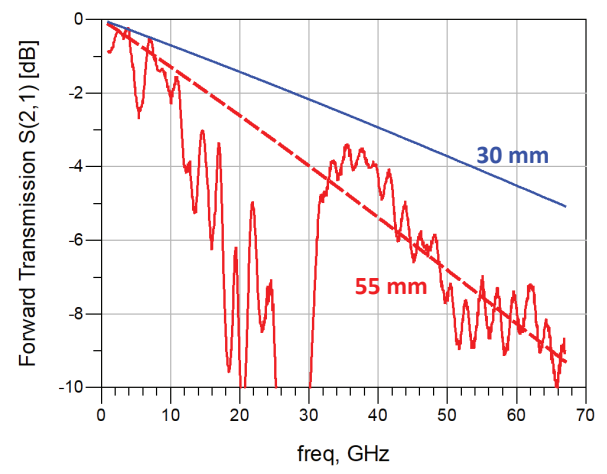

Fig. 3. Forward transmission though the Measured (solid) and extracted (dashed) $55 \mathrm{~mm}$ and $30 \mathrm{~mm}$ textile microstrip lines, up to $67 \mathrm{GHz}$.

a dark-film mask. A minimum feature size of $150 \mu \mathrm{m}$ is achieved using standard PCB manufacturing equipment. Fig. 2 (a-g) shows diagrams and photographs of the antenna throughout the fabrication process.

Although the textile substrate in use has been previously characterized at $2.45 \mathrm{GHz}$ in [12], the inhomogeneity of textiles mean that a new two-line characterization [15], of the sample needed to be performed . Two microstrip lines, of computed $50 \mathrm{ohm}$ impedance were built and measured. The insertion loss $\left(S_{21}\right)$ of a 30 and $55 \mathrm{~mm}$ microstrip on a $310 \mu \mathrm{m}$ woven polyester substrate is shown in Fig. 3, the extracted lines exclude the impedance mismatch losses due to the test connector. The calculated dielectric constant of the full structure, in Fig. 2-d, is 1.64, the measured insertion loss is $0.90 \mathrm{~dB} / \mathrm{cm}$ and $1.79 \mathrm{~dB} / \mathrm{cm}$ at $28 \mathrm{GHz}$ and $60 \mathrm{GHz}$ respectively, only $0.11 \mathrm{~dB} / \mathrm{cm}$ higher at $60 \mathrm{GHz}$ than reported in [8]; due to the thicker textile used in this work. The higher insertion losses in Fig. 3, between 15 and $30 \mathrm{GHz}$ are due to reflection at the connector-VNA plane, due to the low thickness and flexibility of the substrate.

\section{Antenna Simulation and Measurements}

\section{A. Full-Wave Simulation}

A 3D model of the antenna has been created in CST Microwave Studio. A model of the antenna in space, as well as at $1 \mathrm{~mm}$ of CST's Voxel human skin were simulated. As mmWaves do not penetrate past the skin layer, the model only included skin for simplicity [16]. The model, excited using a $50 \mathrm{ohm}$ waveguide port, is shown in Fig. 4. A model of the soldered $1.85 \mathrm{~mm}$ connector has been included in the simulation; to account for the connector-induced variations in the $S_{11}$ measurements, and for de-embedding and correcting the measured s-parameters. The simulated return loss, without the connector, is shown in Fig. 5 exhibiting a $9.5 \mathrm{GHz}$ bandwidth covering the bands of interest.

\section{B. Antenna Measurements}

The antenna under test (AUT) has been tested using an E8361A $67 \mathrm{GHz}$ PNA network analyzer. A $1.85 \mathrm{~mm}$ solderterminated edge launch connector has been used to feed 


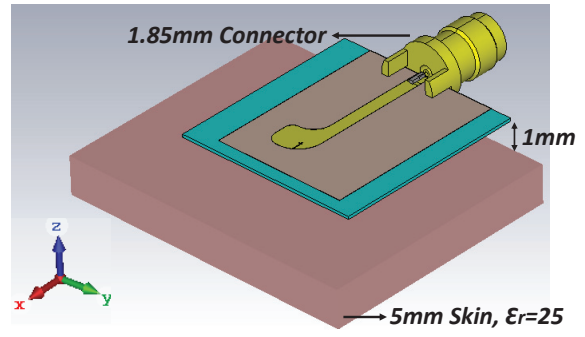

Fig. 4. 3D CST model of the antenna, showing a 5mm-thick skin layer at $1 \mathrm{~mm}$ from the antenna.

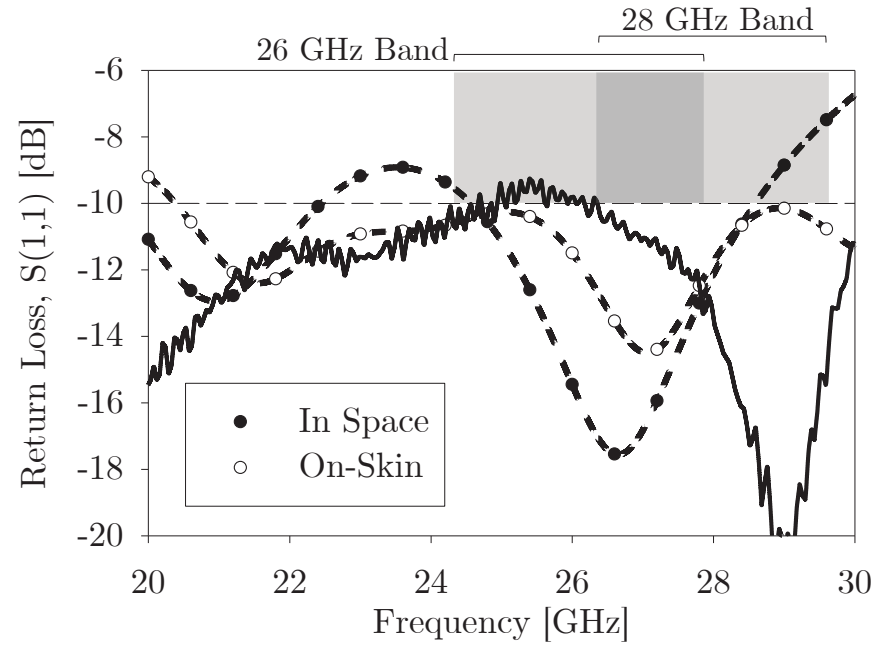

Fig. 5. Simulated (dashed) and measured (solid) $S_{11}$ of the antenna in space and in human proximity.

TABLE I

Simulated And Measured Antenna CHARACTERISTICS

\begin{tabular}{cccc}
\hline & Sim. Gain & Meas. Rlz. Gain & Sim. Rad. Eff. \\
\hline 26 GHz: Space & 5.4 & - & $76.9 \%$ \\
\hline 26 GHz: On-Body & 6.2 & - & $49.3 \%$ \\
\hline 28 GHz: Space & 5.5 & 5.2 & $77.5 \%$ \\
\hline 28 GHz: On-Body & 7.0 & 6.6 & $53.5 \%$ \\
\hline
\end{tabular}

the AUT. The simulated and measured return loss of the antenna including the connector, shown in Fig. 5, exhibit good agreement in the band of interest; validating the simulation approach. The measured s-parameters of the antenna have been corrected, using the 3D connector model to remove the additional reflection. No difference has been observed in the measured $S_{11}$ on and off-body, with and without bending.

The radiation patterns were simulated using far-field monitors in CST at $26 \mathrm{GHz}$ and $28 \mathrm{GHz}$, excited using a $50 \mathrm{ohm}$ discrete port. The gain has been measured by using two AUTs for transmission and reception, and calculating the path losses using the Friis model. The simulated gain and efficiency, as well as measured realized gain, are presented in Table I, details of on-body tuning are in the next subsection. The simulated $3 \mathrm{D}$ and Cartesian gain plots, on- and off-skin, of the antenna are shown in Fig. 6. (a)

(b)

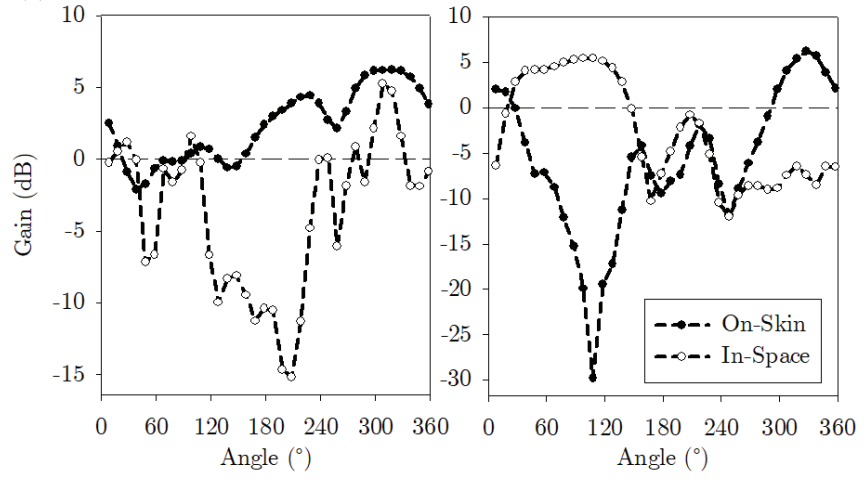

(c)

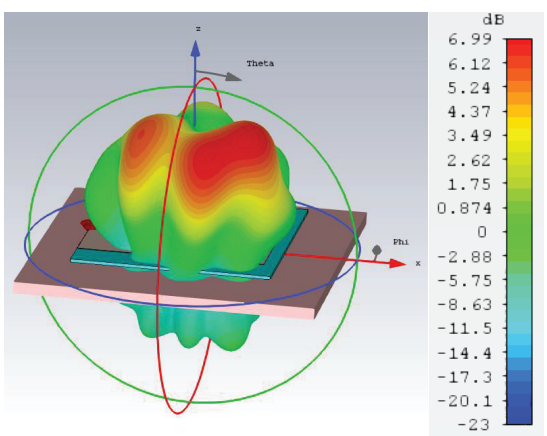

Fig. 6. Antenna computed radiation plots at $28 \mathrm{GHz}$ : E-plane (a), H-plane (b), On-skin 3D pattern (c).
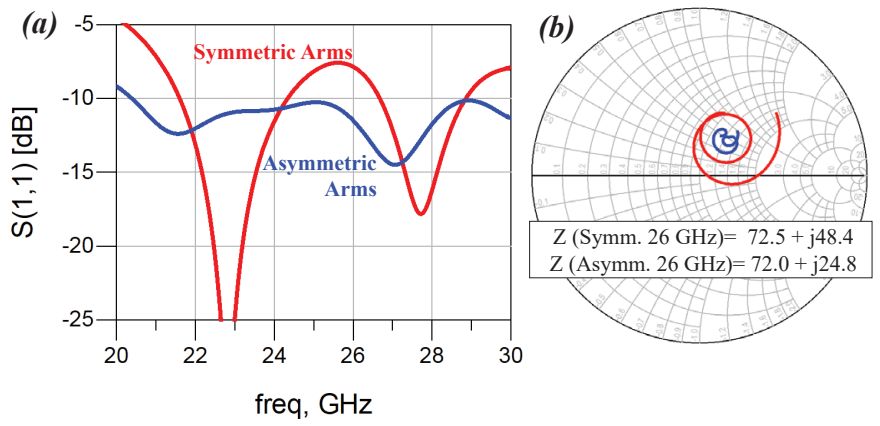

Fig. 7. Simulated $S_{11}$ of the traditional antipodal Vivaldi (red) and the proposed asymmetric antipodal Vivaldi (blue), showing improved bandwidth in (A: $\mathrm{dB}$ magnitude) and a more stable imaginary impedance in (B: Smith chart).

\section{Asymmetry: Overcoming Human-Proximity Effects}

The on-skin antenna experiences additional capacitance due to the higher dielectric constant of skin compared to air, therefore, a highly capacitive component of the antenna's impedance can be observed in the Smith chart in Fig. 7-b. An asymmetric placement of the antipodal arms is proposed, as seen in Fig. 1, in order to reduce the parallel-plate capacitance of the antenna in human proximity. It is seen from Fig. 7-b that the asymmetric placement reduced the imaginary component by $50 \%$ at $26 \mathrm{GHz}$ without resorting to modifying the antenna design. Using the described configuration, the antenna achieves an impedance bandwidth between 20 and 


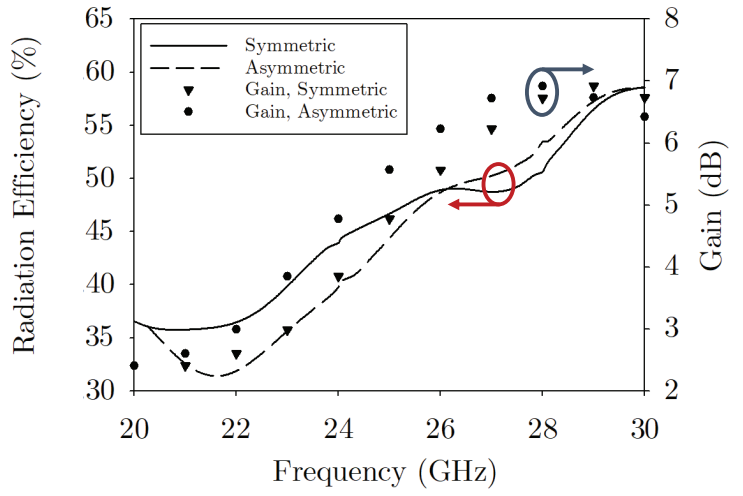

Fig. 8. Simulated peak antenna gain and radiation efficiency, on-skin, of the traditional antipodal Vivaldi and the proposed asymmetric placement.

$30 \mathrm{GHz}$ on-body. Fig. 7-a shows the return loss $\left(S_{11}\right)$ of the antenna with symmetric and asymmetric antipodal arms.

The asymmetric placement of the antenna arms increases the exposure to the human skin beneath the antenna. Although this is expected to reduce the shielding of the antenna, compared to solid-ground-plane antennas (such as patches), a higher gain has been achieved due to the increased reflection of the incident or radiated mmWaves off the skin surface, in the bands of interest. A $1 \mathrm{~dB}$ gain improvement is observed at $26 \mathrm{GHz}$ with a peak efficiency improvement of $5 \%$, Fig. 8 shows the peak on-skin gain and radiation efficiency over the antenna's bandwidth, demonstrating higher gain than previously reported textile mmWave antenna [8].

\section{CONCLUSION}

The first textile UWB on-body antenna for the 5G 26 and 28 $\mathrm{GHz}$ mmWave band is presented, with improved on-body gain and bandwidth, using a novel gain-improvement technique. A simple fabrication process, using standard commercial materials, has been applied to produce a mmWave antenna on textile for RFEH, enabling high efficiency reception of mmWave radiation for wearable rectennas. Future work includes developing microstrip rectifier matching networks on textile, as well as an investigation into minimizing material-induced losses in the mmWave bands.

\section{REFERENCES}

[1] T. A. Khan, A. Alkhateeb, and R. W. Heath, "Millimeter wave energy harvesting," IEEE Transactions on Wireless Communications, vol. 15, 9, pp. $6048-6062,2016$.

[2] I. A. Hemadeh, K. Satyanarayana, M. El-Hajjar, and L. Hanzo, "Millimeter-wave communications: Physical channel models, design considerations, antenna constructions, and link-budget," IEEE Соттиnications Surveys and Tutorials, vol. 20, 2, pp. 870 - 913, 2017.

[3] T. D. P. Perera, D. N. K. Jayakody, S. K. Sharma, S. Chatzinotas, and J. Li, "Simultaneous wireless information and power transfer (swipt): Recent advances and future challenges," IEEE Communication Surveys and Tutorials, vol. 20, 1, pp. $264-302,2018$.

[4] S. Ladan, A. B. Guntupalli, and K. Wu, "A high-efficiency $24 \mathrm{ghz}$ rectenna development towards millimeter-wave energy harvesting and wireless power transmission," IEEE Transactions On Circuits And Systems, vol. 61, 12, pp. 3358 - 3366, 2014
[5] J. Bito, V. Palazzi, J. Hester, R. Bahr, F. Alimenti, P. Mezzanotte, L. Roselli, and M. M. Tentzeris, "Millimeter-wave ink-jet printed rf energy harvester for next generation flexible electronics," in 2017 IEEE Wireless Power Transfer Conference (WPTC), 2017.

[6] A. Collado and A. Georgiadis, "24 ghz substrate integrated waveguide (siw) rectenna for energy harvesting and wireless power transmission," in IEEE MTT-S International Microwave Symposium Digest (MTT), 2013.

[7] S. Harmer, N. Rezgui, N. Bowring, Z. Luklinska, and G. Ren, "Determination of the complex permittivity of textiles and leather in the 14-40 ghz millimetre-wave band using a free-wave transmittance only method," IET Micowave, Antennas, and Propagation, vol. 2, 6, pp. 606 $-614,2008$

[8] N. Chahat, M. Zhadobov, S. A. Muhammad, L. L. Coq, and R. Sauleau, "60-ghz textile antenna array for body-centric communications," IEEE Transactions on Antennas and Propagation, vol. 61, 4, pp. 1816 - 1824, 2013.

[9] L. Alonso-Gonzlez, S. Ver-Hoeye, C. Vzquez-Antua, M. FernndezGarca, and F. L.-H. Andrs, "On the techniques to develop millimeterwave textile integrated waveguides using rigid warp threads," IEEE Transactions on Microwave Theory and Techniques, vol. 66, 2, pp. 751 $-761,2018$.

[10] M. Moosazadeh, S. Kharkovsky, J. T. Case, and B. Samali, "Improved radiation characteristics of small antipodal vivaldi antenna for microwave and millimeter-wave imaging applications," IEEE Antennas and Wireless Propagation Letters, vol. 16, pp. 1961 - 1964, 2017.

[11] B. S. Cook, B. Tehrani, J. R. Cooper, and M. M. Tentzeris, "Multilayer inkjet printing of millimeter-wave proximity-fed patch arrays on flexible substrates," IEEE Antennas and Wireless Propagation Letters, vol. 12, pp. $1351-1354,2013$.

[12] S.-E. Adami, P. Proynov, G. S. Hilton, G. Yang, C. Zhang, D. Zhu, Y. Li, S. P. Beeby, I. J. Craddock, and B. H. Stark, "A flexible 2.45-ghz power harvesting wristband with net system output from $-24.3 \mathrm{dbm}$ of rf power," IEEE Transactions on Microwave Theory and Techniques, 2018.

[13] M. Wagih, Y. Wei, and S. Beeby, "Flexibe $2.4 \mathrm{ghz}$ sensor node for body area networks with a compact high-gain planar antenna," IEEE Antennas and Wireless Propagation Letters, vol. 17, 12, 2018.

[14] K. Yang, R. Torah, Y. Wei, S. Beeby, and J. Tudor, "Waterproof and durable screen printed silver conductive tracks on textiles," Textile Research Journal, vol. 83, pp. 2023 - 2031, 2013.

[15] M.-Q. Lee and S. Nam, "An accurate broadband measurement of substrate dielectric constant," IEEE Microwave and Guided Wave Letters, vol. 6, 4, pp. $168-170,1996$.

[16] A. R. Guraliuc, M. Zhadobov, O. D. Sagazan, and R. Sauleau, "Solid phantom for body-centric propagation measurements at $60 \mathrm{ghz}$," IEEE Transactions on Microwave Theory and Techniques, vol. 62, 6, pp. 1373 - 1380, 2014. 